\title{
Analytical Solution for Elliptical Cloaks Based on the Frequency Selective Surface
}

\author{
Ensieh Ghasemi Mizuji ${ }^{1}$, Ali Abdolali ${ }^{1}$, Iman Derafshi ${ }^{1}$, \\ ${ }^{1}$ Department of Electrical Engineering, Iran University of Science and Technology
}

\begin{abstract}
In this paper the elliptical dielectric cylinder which is covered with FSS is considered. Frequency selective surface cloak which Alu named it mantle cloak is one of the recent techniques for cloaking. In this method an appropriate FSS acts as cloaking device for suppressing the scattering of object in the desired frequency. With using this method the dimension of the cloaks is extremely reduced. By this proposed structure, the RCS of elliptical cylinder decreased about 10-20 $\mathrm{dB}$ therefor designed cloak has an appropriate performance. The analytical solution for the wave in each layer is presented and with using simulation, the electric field and the scattering pattern is drawn.
\end{abstract}

\section{Introduction}

Clocking an object is noticed nowadays. One of the most successful accomplishments for achieving invisibility cloak is based on metamaterials and coordinate transformation [19]. In 2005 plasmonic cloak was invented which was the first step for cloaking, based on suppressing the scattering from the object by Alu and Engeta. In 2009 Alu debated on the new method named mantle cloak for the first time in which the frequency selective surface cancels the scattering from the object [10]. In 2011 according to this idea, the new method for cloaking the planer, cylindrical and spherical objects developed [7]. At the same time the new technique for cloaking, using graphene, is invented [12].

The frequency selective surface is the array of same elements that are infinite in one or two dimensions. The main idea of mantle cloaks is matching the impedance between the object and free space [10-12].

FSS cloak decreases the dimension of the cloaks to one thin surface. Also, it has easier implementation than other methods so it is valuable to practice this method. In this paper the elliptical dielectric covered with frequency selective surface is simulated. The close form for the waves in each layer is presented and the electric and scattering pattern is drawn.

\section{A brief explanation on monolayer cloaks}

In this design, the monolayer FSS with special pattern is used to suppress the scattering of the object. These surfaces which are very smaller than the wavelength in the dimension can be characterized by the surface reactance Zs which includes the real part Rs and the imaginary part Xs that related to surface losses and stored energy respectively
[10-12].

The surface reactance needed to achieve cloaking performance in RF (radio frequency) and FIR (far-infrared) frequencies is created by frequency selective surfaces $[10,11]$ and in $\mathrm{THz}$ band is created using graphene [12].

Similar to plasmonic cloak, this technique is based on scattering cancelation but the difference is that it doesn't require negative polarization. Here the electric field induces the current which cancels the field in opposite phase [1012]. Figure 1 shows the structures simulated in this study.

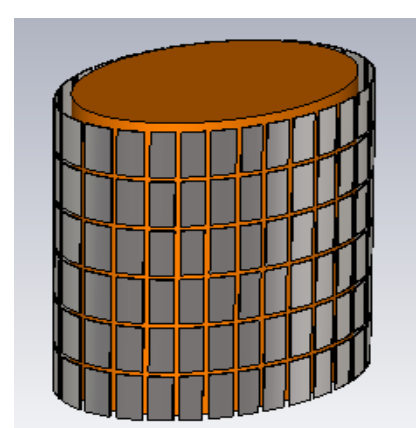

a

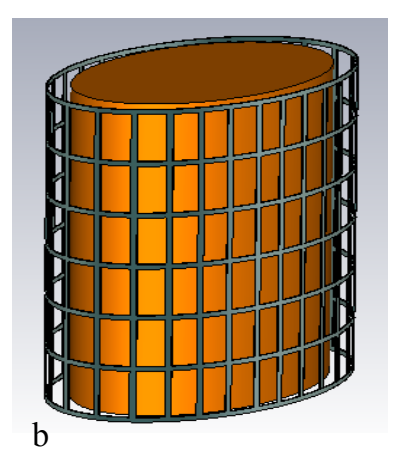

b

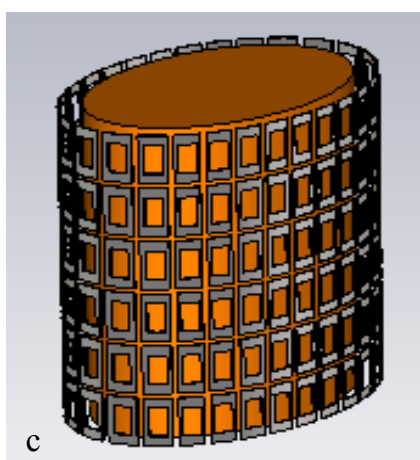

Figure 1: Simulated cloak structures. a) patch structure b) mesh structure c) ring structure.

Bending the FSS creates some coupling capacitors between two surfaces, figure 2 shows this effect. This bending capacitor may change the resonance frequency of the meta surface but according to [] if the element size of the covering FSS be sub-wave length this effect can be ignored. 


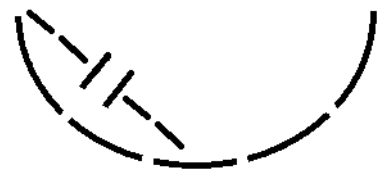

Figure 2: The bending effect.

\section{The wave in the elliptical coordinate}

Picture 3 shows the elliptical coordinate.

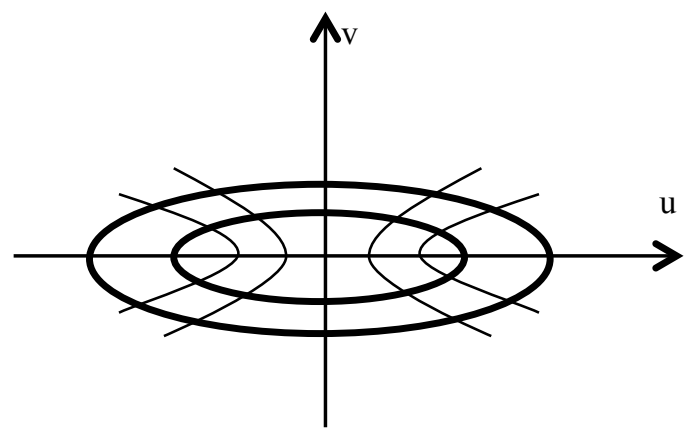

Figure 3: Elliptical coordinate.

The relations between elliptical coordinate $(\mathrm{u}, \mathrm{v}, \mathrm{z})$ and cylindrical coordinate is denoted by equations 1 and 2 :

$$
\begin{aligned}
& \mathrm{x}=\mathrm{d} \cdot \cosh (\mathrm{u}) \cos (\mathrm{v}), \\
& \mathrm{y}=\mathrm{d} \cdot \sinh (\mathrm{u}) \sin (\mathrm{v}),
\end{aligned}
$$

Where $\mathrm{d}$ is confocal distance of ellipse. For solving the scattering problem of TM incident wave, the Helmholtz equation must be satisfied. In elliptical coordinates this equation becomes,

$$
\frac{1}{d^{2}\left(\cosh ^{2}(u)-\cos ^{2}(v)\right)}\left(\frac{\partial^{2} E_{z}}{\partial u^{2}}+\frac{\partial^{2} E_{z}}{\partial v^{2}}\right)+
$$

$\frac{\partial^{2} E_{z}}{\partial z^{2}}+k^{2} E_{z}=0$

$\frac{1}{R} \frac{\partial^{2} R}{\partial u^{2}}+c^{2} k^{2} \cosh ^{2}(u)=\lambda+\frac{1}{2} c^{2} k^{2}$

Using the separation variables and assuming $Z(z)=1$, this equation can be written as,

$$
\begin{aligned}
& \frac{1}{R} \frac{\partial^{2} R}{\partial u^{2}}+c^{2} k^{2} \cosh ^{2}(u)=\lambda+\frac{1}{2} c^{2} k^{2}, \\
& c^{2} k^{2} \cos ^{2}(v)-\frac{1}{\phi} \frac{\partial^{2} \phi}{\partial v^{2}}=\lambda+\frac{1}{2} c^{2} k^{2},
\end{aligned}
$$

If we suppose,

$q=\frac{1}{4} c^{2} k^{2}=\frac{c^{2} \omega^{2}}{4 v^{2}}=\left(\frac{k d}{2}\right)^{2}$,

So,

$$
\begin{aligned}
& \frac{\partial^{2} \phi}{\partial v^{2}}-(\lambda+2 q \cos (2 v) \phi=0, \\
& \frac{\partial^{2} R}{\partial u^{2}}-(\lambda-2 q \cosh (2 u)) R=0,
\end{aligned}
$$

Where the solutions of equations (7) and (8) are Mathieu function and modified Mathieu function, respectively [9-11]. Here a TM wave is supposed and expanded in elliptical coordinate as shown in equation 9:

$$
\begin{aligned}
& \frac{E^{i n c}}{2 \pi}= \\
& \sum_{m=0}^{\infty} \frac{j^{m} M_{c m}^{(1)}\left(q_{N+1}, u\right) c e_{m}\left(q_{N+1}, v\right)}{\int_{0}^{2 \pi} c e_{m}\left(q_{N+1}, v\right)^{2} d v},
\end{aligned}
$$

Equation (9) is yielded by assuming $\alpha=90$. The field in each layer is expressed as,

$$
\begin{aligned}
& E_{z}^{i}=\sum_{m=0}^{\infty}\left[e_{m, 1}^{i} M_{c m}^{(1)}\left(q_{i}, u\right)+e_{m, 2}^{i} M_{c m}^{(2)}\left(q_{i}, u\right)\right] \cdot c e_{m}\left(q_{i}, v\right) \\
& +\sum_{m=1}^{\infty}\left[o_{m, 1}^{i} M_{s m}^{(1)}\left(q_{i}, u\right)+o_{m, 2}^{i} M_{s m}^{(2)}\left(q_{i}, u\right)\right] s e_{m}\left(q_{i}, v\right),
\end{aligned}
$$

Where $\mathrm{ce}_{\mathrm{m}}$ and $\mathrm{se}_{\mathrm{m}}$ are odd and even angular Mathieu functions , $\left(\mathrm{M}_{\mathrm{cm}}{ }^{(1)}, \mathrm{M}_{\mathrm{sm}}{ }^{(1)}\right)$ and $\left(\mathrm{M}_{\mathrm{cm}}{ }^{(2)}, \mathrm{M}_{\mathrm{sm}}{ }^{(2)}\right)$ are first order and second order odd and even radius Mathieu function respectively. The geometry that we should solve as scattering problem is shown in figure 4 .

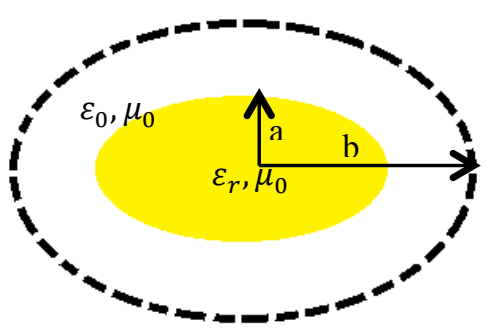

Figure 4: The schematic of our problem. The central ellipse is dielectric and the covering FSS is drawn by dash line.

In outer region the wave is the summation of incident and scattered waves. The scattered is expressed by forth order Mathieu functions $\mathrm{M}_{\mathrm{cm}}{ }^{(4)}$ and $\mathrm{M}_{\mathrm{sm}}{ }^{(4)}[13-15]$. The forth order Mathieu functions act as Hankel functions in cylindrical coordinate.

$$
\begin{aligned}
& E_{z}^{s c a t}=\sum_{m=0}^{\infty} e_{m}^{N+1} M_{c m}^{(4)}\left(q_{N+1}, u\right) c e_{m}\left(q_{N+1}, v\right)+ \\
& \sum_{m=0}^{\infty} o_{m}^{N+1} M_{s m}^{(4)}\left(q_{N+1}, u\right) s e_{m}\left(q_{N+1}, v\right),
\end{aligned}
$$

For determining the magnetic field, below equations can be used [9-11].

$$
\begin{aligned}
& H(u, v)=H_{u}(u, v) \cdot u+H_{v}(u, v) \cdot v= \\
& -\frac{j}{\omega \mu_{i} h}\left(u \frac{\partial}{\partial v} E_{z}(u, v)+v \frac{\partial}{\partial u} E_{z}(u, v)\right), \\
& h=d \sqrt{\cosh ^{2}(u)-\cos ^{2}(v)},
\end{aligned}
$$

For finding unknown coefficients boundary conditions must be applied. at $\mathrm{u}=\mathrm{a}$ electric and magnetic fields are continuous and at $\mathrm{u}=\mathrm{b}$ the electric field is continuous and magnetic field satisfies equation (14)[10-12].

$H_{\text {tan }}{ }^{+}-H_{\text {tan }}{ }^{-}=E_{z} / Z_{s}$, 
By applying the boundary conditions and solving the equations fields in each layer are achieved. For calculating radar cross section versus wave length equation (15) is used,

$\sigma(v)=\lim _{u \rightarrow \infty}\left[2 \pi u \frac{\left|E^{\text {scat }}(u, v)\right|^{2}}{\left|E^{i n c}(u, v)\right|^{2}}\right.$,

And forth order Mathieu function is approximated as (16)[13]:

$$
\begin{aligned}
& M c_{m}^{(4)} \cong \sqrt{\frac{2}{\pi d k \cdot \cosh (u)}} e^{-j\left\{d k \cdot \cosh (u)-\frac{2 m+1}{4} \pi\right\}} \\
& \cong M s_{m}^{(4)}(q, u),
\end{aligned}
$$

Finally scattering width is calculated as 17 :

$$
\begin{aligned}
& \sigma(v)=\frac{4}{k_{0}} \mid \sum_{m=0}^{M} e_{m}^{3} c e_{m}\left(q_{2}, v\right) e^{j\left(\frac{m}{2}\right) \pi} \\
& +\left.\sum_{m=1}^{M} o_{m}^{3} s e_{m}\left(q_{2}, v\right) e^{j\left(\frac{m}{2}\right) \pi}\right|^{2},
\end{aligned}
$$

Fig.5 shows scattering width for the object with and without cloak when $\mathrm{q}_{\mathrm{i}}$ is equal to 0 , where the dielectric permeability and a are 0 and landa/10, respectively. Also it is assumed that the dielectric is covered by patch metasurface which causes $4 \mathrm{nH}$ inductance.

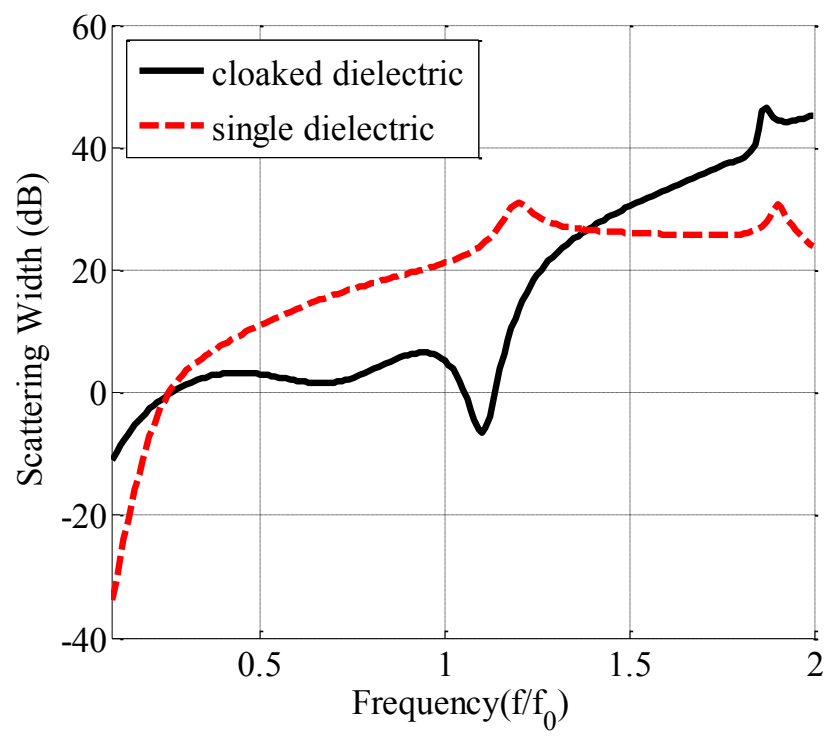

Figure 5: scattering width versus frequency the red line refers to dielectric object without cloak and blue line refers to dielectric with cloak.

As shown in figure 5 the FSS cloak reduces the scattering width in some frequencies more than $20 \mathrm{~dB}$. It means the all waves pass the object and the scattering is suppressed. Using this method in section IV the CST simulation results for an elliptical object is presented.

\section{Simulation results}

In this section the electric field of the elliptical object covered with various meta-surface structures is studied for an incident plane wave. The schematic is given in figure 6 . The amplitude of the electric field for an infinite elliptical object with $\mathrm{a}=0.85 \mathrm{~cm}$ and $\mathrm{b}=1.5 \mathrm{a}$ is plotted in figures $8-\mathrm{b}$, 8 -c and 8-d for $12 \mathrm{GHz}$. The $\mathrm{a}_{\mathrm{c}}=1.25^{*} \mathrm{a}$ and $\mathrm{b}_{\mathrm{c}}=1.25^{*} \mathrm{~b}$ are the radiuses of the elliptical covering FSS. Other dimensions of the FSSs are given in figure 7.

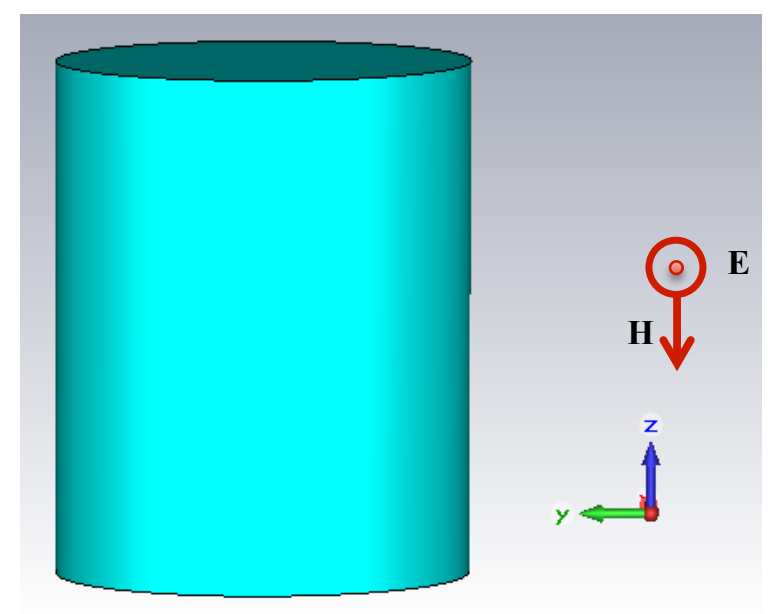

Fig.6. The schematic of the elliptical object.
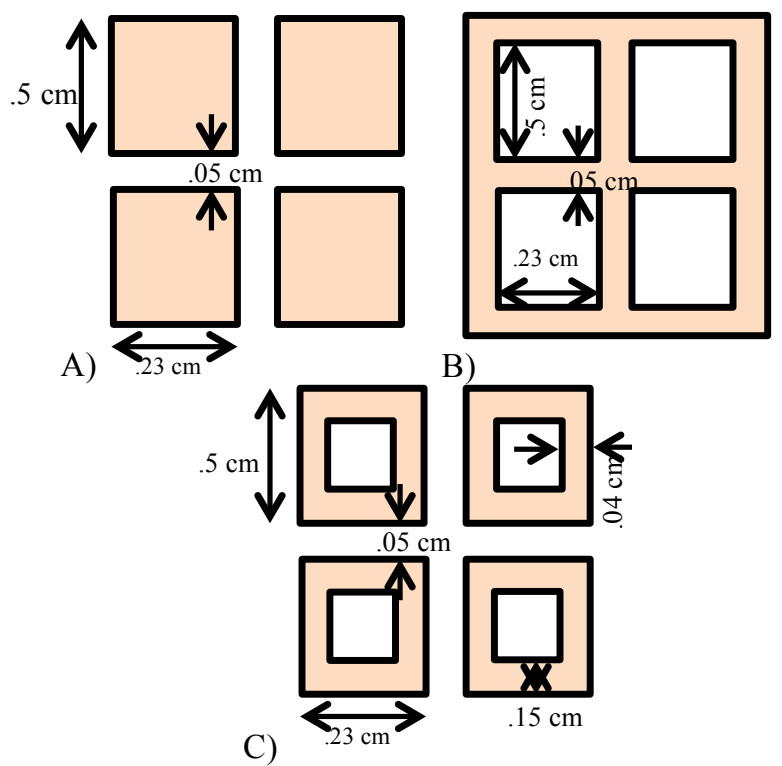

B)

Figure 7: A) patch structure. B) mesh structure. C) ring structure.
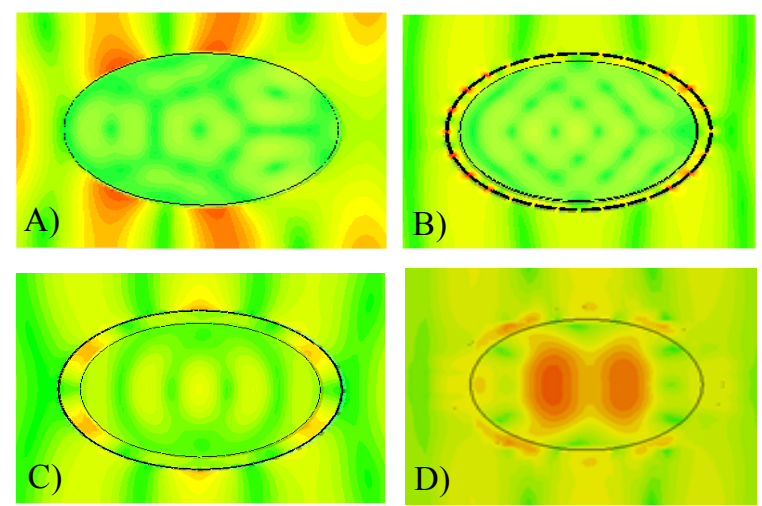
Figure 8: The electric field for A) the object without cloak. B) patch structure. C) mesh structure. D) ring structure.

Figure 9 shows the simulation results for farfield RCS which is found using CST 2012.

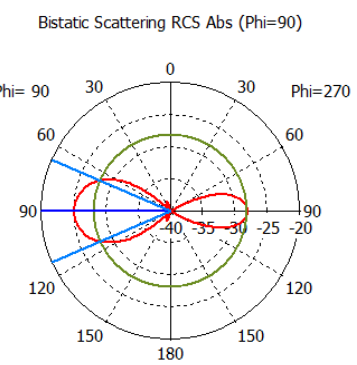

A) Theta / Degree vs. dBm2 Bistatic Scattering RCS Abs (Phi=90)

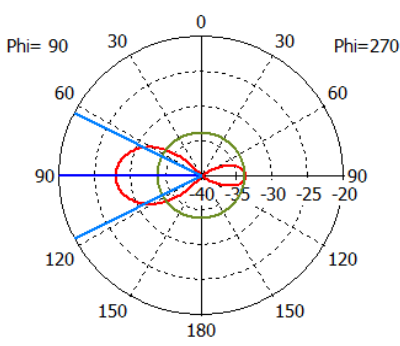

C) Theta / Degree vs. dBm2

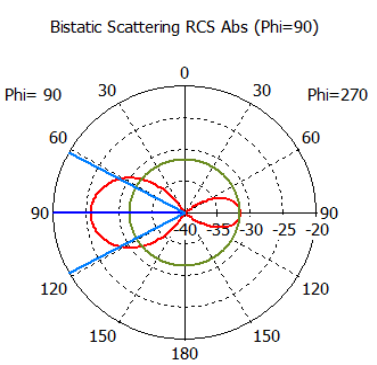

B) Theta / Degree vs. dBm2 Bistatic Scattering RCS Abs (Phi $=90$ )

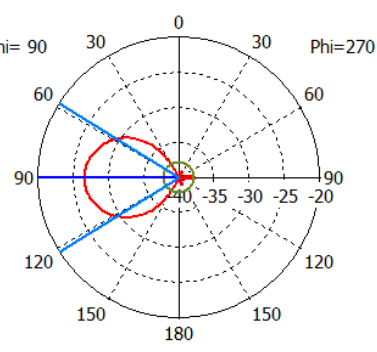

D) Theta / Degree vs. dBm2
Figure 9: The far field scattering pattern a) the object without cloak. b) patch structure. c) mesh structure. d) ring structure.

As it is shown in figure 9 the RCS is reduced when the FSS is utilized for covering the dielectric object. Results in figures 7 and 8 demonstrates adding the FSS cloak suppress the scattering of the object.

\section{Conclusions}

Adding a frequency selective surface is proposed as new method for cloaking which is based on scattering suppression. With using this method the implementation of cloak may be facilitated and the dimension and the volume of cloaks are reduced greatly. In this paper the analytical solution for elliptical FSS cloak is presented and the electric field and farfield scattering pattern are drawn for several structures using CST. This method improves the amount RCS reduction and the cloaking performance. Our design has finite bandwidth; applying multilayered dielectric structure may be useful for increasing the bandwidth. Of course this technique increases the loss. .

\section{References}

[1] B. Zhang, H. Chen, and B.-I. Wu,"Practical Limitations of An Invisibility Cloak", Progress In Electromagnetic Research,407-416,(2009)
[2] Q. Cheng, W. X. Jiang, and T.-J. Cui," Investigations of The Electromagnetic Properties of Three-Dimentinal Arbitrarily-Shaped Cloaks", Progress In Electromagnetic Research,105-117,(2009)

[3] X. Chen," Implicit Boundary Conditions in Transformation-Optics Cloaking for Electromagneticwaves"Progress In Electromagnetic Research,521-534,(2011)

[4] C. F. Yang, M. Huang, J. J. Yang, and Z. Xiao, "An External Cloak With Arbitrary Cross Section Based On Complementary Medium," Progress In Electromagnetics Research M, Vol. 10, 1324, (2009).

[5] B. Zhang, H. Chen, and B.-I. Wu," Practical Limitations of An Invisibility Cloak",Progress In Electromagnetic Research,407-416, (2009).

[6] Q. Cheng, W. X. Jiang, and T.-J. Cui,” Investigations of The Electromagnetic Properties of Three-Dimentinal Arbitrarily-Shaped Cloaks", Progress In Electromagnetic Research,105-117, (2009).

[7] X. Chen," Implicit Boundary Conditions in Transformation-Optics Cloaking for Electromagneticwaves" Progress In Electromagnetic Research, 521-534, (2011).

[8] K. Agarwal, X. Chen, L. Hu, H. Liu, and G. Uhlmann," Polarization-Invariant Directional Cloaking by Transformation Optics", Progress In Electromagnetic Research, 415-423, (2011).

[9] S. Y. Lai, H.Chen, Zhao-Qing Zhang, and C. T. Chan, "Complementary Media Invisibility Cloak that Cloaks Objects at a Distance Outside the Cloaking" physical review letters, 102, 093901, (2009).

[10] Andrea Alù, "Mantle cloak: Invisibility induced by a surface", physical review B 80, 245115, (2009).

[11] Pai-Yen Chen and Andrea Alu, "Mantle cloaking using thin patterned metasurfaces", physical review B 84, 205110 , (2011).

[12] Pai-Yen Chen and Andrea Alu, "Atomically Thin Surface Cloak Using Graphene”, ACSnano, 5855-5863, (2011).

[13] E. Cojocaru, "Elliptic Cylindrical Invisibility Cloak, a Semianalytical Approach Using Mathieu Functions", physics, (2008). 
[14] S.Caorsi, M.Pastorino and M.Raffetto," Scattering by a conducting elliptic cylinder with a multilayer dielectric coating", Radio Science, p-p 2155-2166, NovemberDecember, (1998).

[15] Salvatore Caorsi, Matteo Pastorino and Mirco Raffetto," Electromagnetic Scattering by a Multilayer Elliptic Cylinder Under Transverse-Magnetic Illumination: Series Solution in Terms of Mathieu Functions", IEEE TRANSACTIONS ON ANTENNAS AND PROPAGATION, VOL. 45, NO. 6, JUNE (1997).

[16] Jc Soric, P.Y. Chen, A. Kerkhoff, D. Rainwater, K. Melin and Alu, Demonstration of ultralow profile cloak for scattering suppression of a finite-length rod in free space", New journal of Physics, volum 15, March (2013). 